\title{
Characterizations of Exponentiated Distributions
}

Gholam Hossein Hamedani

Marquette University

United States of America

gholamhossein.hamedani@mu.edu

\section{Abstract}

Various characterizations of the class of exponentiated distributions are presented. These characterizations are based on a simple relationship between two truncated moments and based on functions of the $n^{\text {th }}$ order statistic. The results are applied to certain well-known members of this class.

\section{Introduction}

The problem of characterizing a distribution is an important problem which has recently attracted the attention of many researchers. Thus, various characterizations have been established in many different directions.

Recently, the $\mathrm{F}^{\alpha}$-distributions (or exponentiated distributions, defined below) have been shown to have a wide domain of applicability, in particular in modeling and analysis of life time data. Let $F$ be an absolutely continuous cdf (cumulative distribution function) with support on $(a, b)$, where the interval may be unbounded, and let $\alpha$ be a positive real number. The random variable $X$ has an $F^{\alpha}$-distribution if its $\mathrm{cdf}$, denoted by $F_{x}$, is given by $F_{x}(x)=F^{\alpha}(x)=[F(x)]^{\alpha}$. The class of $F^{\alpha}$-distributions contains certain well-known distributions for which their cdf ' $s$ have closed forms (see, e.g. Gupta et al. (1998), Gupta and Kundu (1999, 2000, 2001, 2007) and Nadarajah (2011)). For further details and the domain of applicability of this class, we refer the interested reader to the above mentioned articles and the most recent work of Shakil and Ahsanullah (2012). In the statistical applications where the underlying distribution is assumed to be an $F^{\alpha}$-distribution, the investigator needs to verify that this distribution is in fact the desired $F^{\alpha}$-distribution. To this end the investigator has to rely on the characterizations of the underlying distribution and determine if the corresponding conditions are satisfied. So, the problem of characterizing $F^{\alpha}$-distribution becomes essential. Our objective here, is to present characterizations of $F^{\alpha}$-distributions. We shall do this in two different directions as discussed in Section 2 below.

The pdf (probability density function), $f_{x}$, of an $F^{\alpha}$-distribution, $F_{x}$, with base cdf $F$, is given by

$$
\mathrm{f}_{\mathrm{x}}(\mathrm{x})=\alpha \mathrm{f}(\mathrm{x})(\mathrm{F}(\mathrm{x}))^{\alpha-1}, \mathrm{a}<\mathrm{x}<\mathrm{b},
$$

where $f$ is the pdf corresponding to the cdf $F$. 


\section{Characterization R esults}

As we mentioned in the previous section, the $F^{\alpha}$-distributions have applications in many fields of study, which have been mentioned in Shakil and Ahsanullah (2012) and the references therein. So, an investigator will be vitally interested to know if their model fits the requirements of $F^{\alpha}$-distribution. To this end, the investigator relies on characterizations of this distribution, which provide conditions under which the underlying distribution is indeed an $\mathrm{F}^{\alpha}$-distribution. In this section we will present various characterizations of the $\mathrm{F}^{\alpha}$-distributions.

Throughout this section we assume, when necessary, that the base distribution $F$ is twice differentiable on its support.

\subsection{Characterization based on two truncated moments}

In this subsection we present characterizations of the $F^{\alpha}$-distributions in terms of truncated moments. We like to mention here the works of Galambos and Kotz (1978), Kotz and Shanbhag (1980), Glänzel (1988, 1987 and 1990), Glänzel et al. (1994), Glänzel et al. (1984), Glänzel and Hamedani (2001) and Hamedani (1993, 2002 and 2006) in this direction. Our characterization results presented here will employ an interesting result due to Glänzel (1987) (Theorem G below).

Theorem $G$. Let $(\Omega, P)$ be a given probability space and let $H=[a, b]$ be an interval for some $a<b \quad(a=-\infty, b=\infty$ mightaswellbeallowed $)$. Let $X: \Omega \rightarrow H \quad$ be a continuous random variable with the distribution function $F$ and let $g$ and $h$ be two real functions defined on $H$ such that

$$
\mathrm{E}[\mathrm{g}(\mathrm{X}) \mid \mathrm{X} \geq \mathrm{X}]=\mathrm{E}[\mathrm{h}(\mathrm{X}) \mid \mathrm{X} \geq \mathrm{x}] \eta(\mathrm{x}), \quad \mathrm{X} \in \mathrm{H},
$$

is defined with some real function $\eta$. Assume that $g, h \in \mathrm{C}^{1}(\mathrm{H}), \eta \in \mathrm{C}^{2}(\mathrm{H})$ and $\mathrm{F}$ is twice continuously differentiable and strictly monotone function on the set $\mathrm{H}$. Finally, assume that the equation $h \eta=g$ has no real solution in the interior of $H$. Then $\mathrm{F}$ is uniquely determined by the functions $\mathrm{g}, \mathrm{h}$ and $\eta$, particularly

$$
F(x)=\int_{a}^{x} c\left|\frac{\eta^{\prime}(u)}{\eta(u) h(u)-g(u)}\right| \exp (-s(u)) d u,
$$

where the function $s$ is a solution of the differential equation $s^{\prime}=\frac{\eta^{\prime} h}{\eta h-g}$ and $C$ is a constant, chosen to make $\int_{H} d F=1$.

Remarks 2.1.1. (a) In Theorem G, the interval $H$ need not be closed. (b) The goal is to have the function $\eta$ as simple as possible. For a more detailed discussion on the choice of $\eta$, we refer the reader to Glänzel and Hamedani (2001) and Hamedani (1993, 2002 and 2006). 
Proposition 2.1.2. Let $\mathrm{X}: \Omega \rightarrow(\mathrm{a}, \mathrm{b})$ be a continuous random variable, $\mathrm{F}$ an absolutely continuous cdf with pdf $f$ and let $h(x)=1$ and $g(x)=\int_{a}^{x}$ $f(u)(F(u))^{\alpha-1} d u$ for $x \in(a, b)$. The pdf of $X$ is (1.1) if and only if the function $\eta$ defined in Theorem $\mathrm{G}$ has the form

$$
\left.\eta(\mathrm{x})=\frac{1}{2 \alpha}+\frac{1}{2} \int_{\mathrm{a}}^{\mathrm{x}} \mathrm{f}(\mathrm{u})(\mathrm{F}(\mathrm{u}))^{\alpha-1} \mathrm{du}\right), \mathrm{a}<\mathrm{x}<\mathrm{b} .
$$

Proof. Let $X$ have pdf (1.1), then

$$
\left(1-F_{x}(x)\right) E[h(X) \mid X \geq x]=\left(1-F_{x}(x)\right), \quad a<x<b,
$$

and

$$
\begin{aligned}
& \left(1-F_{x}(x)\right) E[g(X) \mid X \geq x] \\
= & \int_{x}^{b} \int_{a}^{t} f(u)(F(u))^{\alpha-1} d u f_{x}(t) d t \\
= & \int_{x}^{b} \frac{1}{\alpha} F_{x}(t) f(t) d t=\frac{1}{2 \alpha}\left(1-\left(F_{x}(x)\right)^{2}\right), a<x<b,
\end{aligned}
$$

and finally

$$
\eta(x) h(x)-g(x)=\frac{1}{2}\left(\frac{1}{\alpha}-\int_{a}^{x} f(u)(F(u))^{\alpha-1} d u\right) \neq 0 \text { for } a<x<b .
$$

Conversely, if $\eta$ is given as above, then

$$
s^{\prime}(\mathrm{x})=\frac{\eta^{\prime}(\mathrm{x}) \mathrm{h}(\mathrm{x})}{\eta(\mathrm{x}) \mathrm{h}(\mathrm{x})-\mathrm{g}(\mathrm{x})}=\frac{\mathrm{f}(\mathrm{x})(\mathrm{F}(\mathrm{x}))^{\alpha-1}}{\left(\frac{1}{\alpha}-\int_{\mathrm{a}}^{\mathrm{x}} \mathrm{f}(\mathrm{u})(\mathrm{F}(\mathrm{u}))^{\alpha-1} \mathrm{du}\right)},
$$

and hence

$$
s(x)=-\ln \left(\frac{1}{\alpha}-\int_{a}^{x} f(u)(F(u))^{\alpha-1} d u\right), a<x<b .
$$

Now, in view of Theorem $\mathrm{G}$ (with $\mathrm{C}=\alpha$ ), $\mathrm{X}$ has pdf (1.1).

Corollary 2.1.3. Let $X: \Omega \rightarrow(a, b)$ be a continuous random variable and let $h(x)=1$ for $x \in(a, b)$. The pdf of $X$ is (1.1) if and only if there exist functions $g$ and $\eta$ defined in Theorem $\mathrm{G}$ satisfying the differential equation

$$
\frac{\eta^{\prime}(\mathrm{x})}{\eta(\mathrm{x})-\mathrm{g}(\mathrm{x})}=\frac{\mathrm{f}(\mathrm{x})(\mathrm{F}(\mathrm{x}))^{\alpha-1}}{\left(\frac{1}{\alpha}-\int_{\mathrm{a}}^{\mathrm{x}} \mathrm{f}(\mathrm{u})(\mathrm{F}(\mathrm{u}))^{\alpha-1} \mathrm{du}\right)}, \quad \mathrm{a}<\mathrm{x}<\mathrm{b} .
$$

Remark 2.1.4. The general solution of the differential equation given in Corollary 2.1.3 is

$$
\eta(\mathrm{x})=\left(\frac{1}{\alpha}-\int_{\mathrm{a}}^{\mathrm{x}} \mathrm{f}(\mathrm{u})(\mathrm{F}(\mathrm{u}))^{\alpha-1} \mathrm{du}\right)^{-1}\left[-\int \mathrm{g}(\mathrm{x}) \mathrm{f}(\mathrm{x})(\mathrm{F}(\mathrm{x}))^{\alpha-1} \mathrm{dx}+\mathrm{D}\right]
$$


for $a<x<b$, where $D$ is a constant. One set of appropriate functions is given in Proposition 2.1.2 with $\mathrm{D}=\frac{1}{2 \alpha^{2}}$.

Remark 2.1.5. Due to the generality of the expression for the $\mathrm{F}^{\alpha}$-distributions (being a power of a given base cdf ), the statement of Proposition 2.1.2 is given in a general form in which we assume $h(x)=1$ for $x \in(a, b)$. We will, however, take different $h(x)$ and $g(x)$ for the special cases that we characterize below in the interest of having $\eta(\mathrm{x})$ as simple as possible.

(I) Generalized Exponential (GE) or Exponentiated Exponential (EE) Distribution.

The cdf and pdf for this subclass of $F^{\alpha}$-distributions are given, respectively, by

$$
\begin{aligned}
& F_{x}(x)=[F(x)]^{\alpha}=\left(1-e^{-\lambda x}\right)^{\alpha}, x \geq 0, \\
& f_{x}(x)=\alpha \lambda e^{-\lambda x}\left(1-e^{-\lambda x}\right)^{\alpha-1}, x>0,
\end{aligned}
$$

where $\lambda, \alpha$ are positive parameters.

Proposition 2.1.2 with $\quad h(x)=\left(1-e^{-\lambda x}\right)^{-(\alpha-1)} \quad, \quad g(x)=x\left(1-e^{-\lambda x}\right)^{-(\alpha-1)} \quad$ and $\eta(\mathrm{x})=\mathrm{x}+\frac{1}{\lambda}$, gives a characterization of this subclass of distributions.

(II) Exponentiated Weibull (EW) Distribution.

The cdf and pdf for this subclass of $\mathrm{F}^{\alpha}$-distributions are given, respectively, by

$$
\begin{aligned}
& \mathrm{F}_{\mathrm{x}}(\mathrm{x})=[\mathrm{F}(\mathrm{x})]^{\alpha}=\left(1-\mathrm{e}^{-\lambda \mathrm{x}^{\delta}}\right)^{\alpha}, \mathrm{x} \geq 0, \\
& \mathrm{f}_{\mathrm{x}}(\mathrm{x})=\alpha \lambda \delta \mathrm{x}^{\delta-1} \mathrm{e}^{-\lambda \mathrm{x}^{\delta}}\left(1-\mathrm{e}^{-\lambda \mathrm{x}^{\delta}}\right)^{\alpha-1}, \mathrm{x}>0,
\end{aligned}
$$

where $\lambda, \alpha, \delta$ are positive parameters.

Proposition 2.1.2 with $\quad \mathrm{h}(\mathrm{x})=\left(1-\mathrm{e}^{-\lambda \mathrm{x}^{\delta}}\right)^{-(\alpha-1)} \quad, \quad \mathrm{g}(\mathrm{x})=\mathrm{x}\left(1-\mathrm{e}^{-\lambda \mathrm{x}^{\delta}}\right)^{-(\alpha-1)} \quad$ and $\eta(\mathrm{x})=\mathrm{x}+\frac{\mathrm{e}^{\lambda \mathrm{x}^{\delta}}}{\delta \lambda^{\frac{1}{\delta}}} \Gamma\left(\lambda \mathrm{x}^{\delta} ; \frac{1}{\delta}\right)$ where $\Gamma(\mathrm{u} ; \beta)=\int_{u}^{\infty} \mathrm{v}^{\beta-1} \mathrm{e}^{-\mathrm{v}} \mathrm{dv}$, gives a characterization of this subclass of distributions.

Note that for $\delta=1$ we have the case (I) above and for $\delta=2$, Burr Type X or Generalized Rayleigh (GR) distribution is a special case of (EW). 
(III) Exponentiated Pareto or Lomax (EP or EL) Distribution.

The cdf and $p d f$ for this subclass of $F^{\alpha}$-distributions are given, respectively, by

$$
\begin{aligned}
& \mathrm{F}_{\mathrm{x}}(\mathrm{x})=[\mathrm{F}(\mathrm{x})]^{\alpha}=\mathrm{x}^{\alpha \delta}\left(\mathrm{x}^{\delta}+\lambda\right)^{-\alpha}, \mathrm{x} \geq 0, \\
& \mathrm{f}_{\mathrm{x}}(\mathrm{x})=\alpha \delta \lambda \mathrm{x}^{\alpha \delta-1}\left(\mathrm{x}^{\delta}+\lambda\right)^{-(\alpha+1)}, \mathrm{x}>0,
\end{aligned}
$$

where $\lambda, \alpha, \delta$ are positive parameters.

Let $\gamma$ be an arbitrary positive real number. Proposition 2.1.2 with $\delta>\gamma$, $\mathrm{h}(\mathrm{x})=\mathrm{x}^{-(\alpha+1) \delta}\left(\mathrm{x}^{\delta}+\lambda\right)^{(\alpha+1)}, \quad \mathrm{g}(\mathrm{x})=\mathrm{x}^{-(\alpha+1) \delta+\gamma}\left(\mathrm{x}^{\delta}+\lambda\right)^{(\alpha-1)}, \quad$ and $\quad \eta(\mathrm{x})=\left(\frac{\delta}{\delta-\gamma}\right) \mathrm{x}^{\gamma}$, gives a characterization of this subclass of distributions. Another version of (EP or EL) has cdf $\mathrm{F}_{\mathrm{x}}(\mathrm{x})=\left[1-(1+\mathrm{x})^{-\beta}\right]^{\alpha}, \quad \mathrm{x} \geq 0$ for which $\mathrm{h}(\mathrm{x}), \mathrm{g}(\mathrm{x})$ and $\eta(\mathrm{x})$ can be defined as well.

(IV) Generalized Generalized Logistic (GGL) Distribution.

The cdf and pdf for this subclass of $\mathrm{F}^{\alpha}$-distributions are given, respectively, by

$$
\begin{aligned}
& \mathrm{F}_{\mathrm{x}}(\mathrm{x})=\left(1+\mathrm{e}^{-\lambda \mathrm{x}^{\delta}}\right)^{-\alpha}, \mathrm{x} \in \mathrm{R}, \\
& \mathrm{f}_{\mathrm{x}}(\mathrm{x})=\alpha \delta \lambda \mathrm{x}^{\delta-1} \mathrm{e}^{-\lambda \mathrm{x}^{\delta}}\left(1+\mathrm{e}^{-\lambda \mathrm{x}^{\delta}}\right)^{-(\alpha+1)}, \mathrm{x} \in \mathrm{R},
\end{aligned}
$$

where $\lambda, \alpha>0$ and $\delta$ a positive odd integer, are parameters.

Proposition 2.1.2 with $\quad h(x)=\left(1+e^{-\lambda x^{\delta}}\right)^{(\alpha+1)}, \quad g(x)=x\left(1+e^{-\lambda x^{\delta}}\right)^{(\alpha+1)}, \quad$ and $\eta(\mathrm{x})=\mathrm{x}+\frac{\mathrm{e}^{\lambda x^{\delta}}}{\delta \lambda^{\frac{1}{\delta}}} \Gamma\left(\lambda \mathrm{x}^{\delta} ; \frac{1}{\delta}\right)$, gives a characterization of this subclass of distributions.

For $\delta=1$, the corresponding distribution is called Generalized Logistic (GL), so for $\delta$ a positive odd integer we call the corresponding distribution (GGL).

Remark 2.1.6. Clearly there are other suitable functions $h(x), g(x)$ and $\eta(x)$ for the above special cases (I)-(IV).

2.2. Characterization based on truncated moments of certain functions of the $n^{\text {th }}$ order statistic

Let $X_{1: n} \leq X_{2: n} \leq \ldots \leq X_{n: n}$ be $n$ order statistics from an $F^{\alpha}$-distribution with base cdf $F$. We present here a characterization result for $F^{\alpha}$-distributions based on truncated moments of the $n^{\text {th }}$ order statistic. We refer the reader to Ahsanullah and Hamedani (2007), Hamedani et al. (2008) and Hamedani (2010), among others, for 
characterizations of other well-known continuous distributions in this direction. The proof of the following proposition is similar to that of Theorem 2.5 of Hamedani (2010) in which $\mathrm{k}(\mathrm{x})=\mathrm{x}^{\delta}$ for some $\delta>0$. We give a brief proof of the general $\mathrm{k}(\mathrm{x})$, however, for the sake of completeness.

Proposition 2.2.1. Let $X: \Omega \rightarrow(a, b)$ be a continuous random variable with $F^{\alpha}-$ distribution $F_{X}$ and $k(x)$ be a differentiable function such that $\lim _{x \rightarrow a} k(x)\left(F_{x}(x)\right)^{n}=0$. Let $q(x, n)$ be a real-valued function which is differentiable with respect to $x$ and $\int_{a}^{b} \frac{k^{\prime}(x)}{q(x, n)} d x=\infty$. Then

$$
E\left[k\left(X_{n: n}\right) \mid X_{n: n}<t\right]=k(t)-q(t, n), a<t<b \text {, }
$$

implies that

$$
F_{x}(x)=\left(\frac{q(b, n)}{q(x, n)}\right)^{\frac{1}{n}} \exp \left\{-\int_{x}^{b} \frac{k^{\prime}(t)}{n q(t, n)} d t\right\}, \quad x \geq a .
$$

Proof. Condition (2.2.1) and the assumption $\lim _{x \rightarrow a} k(x)\left(F_{x}(x)\right)^{n}=0$ imply

$$
\int_{a}^{t} k^{\prime}(x)\left(F_{x}(x)\right)^{n} d x=q(t, n)\left(F_{x}(t)\right)^{n} \text {. }
$$

Differentiating (2.2.2) with respect to $t$, we obtain

$$
\frac{f_{x}(t)}{F_{x}(t)}+\frac{\frac{\partial}{\partial t} q(t, n)}{n q(t, n)}=\frac{k^{\prime}(t)}{n q(t, n)} .
$$

Integrating (2.2.3) with respect to $t$, from $x$ to $b$, results in

$$
F_{x}(x)=\left(\frac{q(b, n)}{q(x, n)}\right)^{\frac{1}{n}} \exp \left\{-\int_{x}^{b} \frac{k^{\prime}(t)}{n q(t, n)} d t\right\}, \quad x \geq a .
$$

Remarks 2.2.2. (i) In Proposition 2.2.1, the interval $(a, b)$ is allowed to be unbounded, as we mentioned in the Introduction. (ii) In Hamedani (2010), the author did not have any applications of their Theorem 2.5. We are now pleased to see that there are distributions for which Proposition 2.2.1 (or Theorem 2.5) can be employed to characterize them. We now present characterizations of $\mathrm{F}^{\alpha}$-distributions (I)-(IV) (see our Remark 2.1.5) based on certain functions of the $n^{\text {th }}$ order statistic, $X_{n: n}$. It is rather straightforward to show that the conditions of Proposition 2.2.1 are satisfied in cases (I), (II) and (IV). The case (III) requires rewriting $F_{x}(x)$ in slightly different form $\left(F_{x}(x)=\left(1-\lambda\left(x^{\delta}+\lambda\right)^{-1}\right)^{\alpha}\right)$ and regrouping terms appropriately to show that the conditions as well as conclusion of Proposition 2.2.1 are satisfied. 
(I) For $\mathrm{k}(\mathrm{x})=\left(1-\mathrm{e}^{-\lambda \mathrm{x}}\right)^{\alpha}$ and $\mathrm{q}(\mathrm{x}, \mathrm{n})=\frac{1}{\mathrm{n}+1}\left(1-\mathrm{e}^{-\lambda \mathrm{x}}\right)^{\alpha},(2.2 .1)$ provides a characterization of (GE or EE) distributions. For $k(x)=\left(1-e^{-\lambda x}\right)$ and $q(x, n)=\frac{1}{n \alpha+1}\left(1-e^{-\lambda x}\right), \quad$ (2.2.1) provides a characterization of (GE or EE) distributions as well.

(II) For $\mathrm{k}(\mathrm{x})=\left(1-\mathrm{e}^{-\lambda \mathrm{x}^{\delta}}\right)^{\alpha}$ and $\mathrm{q}(\mathrm{x}, \mathrm{n})=\frac{1}{\mathrm{n}+1}\left(1-\mathrm{e}^{-\lambda \mathrm{x}^{\delta}}\right)^{\alpha}$, (2.2.1) provides a characterization of $(E W)$ distributions. As expected the functions $k(x)=\left(1-e^{-\lambda x^{\delta}}\right)$ and $q(x, n)=\frac{1}{n \alpha+1}\left(1-e^{-\lambda x}\right)$ will work as well.

(III) For $\mathrm{k}(\mathrm{x})=\left(\mathrm{x}^{\delta}+\lambda\right)^{-1}$ and $\mathrm{q}(\mathrm{x}, \mathrm{n})=-\frac{1}{\lambda(\mathrm{n} \alpha+1)} \mathrm{x}^{\delta}\left(\mathrm{x}^{\delta}+1\right)^{-1},(2.2 .1)$ provides a characterization of (EP or EL) distributions.

(IV) For $\mathrm{k}(\mathrm{x})=\left(1+\mathrm{e}^{-\lambda \mathrm{x}^{\delta}}\right)^{-1}$ and $\mathrm{q}(\mathrm{x}, \mathrm{n})=\frac{1}{\mathrm{n} \alpha+1}\left(1+\mathrm{e}^{-\lambda \mathrm{x}^{\delta}}\right)^{-1},(2.2 .1)$ provides a characterization of (GGL) distributions.

\section{Refer ences}

1. Ahsanullah, M. and Hamedani, G.G. (2007), Certain characterizations of the power function and beta distributions based on order statistics, JSTA, 6, $220-225$.

2. Galambos, J. and Kotz, S. (1978), Characterizations of probability distributions. A unified approach with an emphasis on exponential and related models, Lecture Notes in Mathematics, 675, Springer, Berlin.

3. Glänzel, W. (1988), A characterization of the normal distribution, Studia Sci. Math. Hungar. 23, 89-91.

4. Glänzel, W. (1987), A characterization theorem based on truncated moments and its application to some distribution families, Mathematical Statistics and Probability Theory (Bad Tatzmannsdorf, 1986), Vol. B, Reidel, Dordrecht, $75-84$.

5. Glänzel, W. (1990), Some consequences of a characterization theorem based on truncated moments, Statistics 21, 613-618.

6. Glänzel, W., IrWin - (1994). A characterization tool for discrete distributions under Window(R), Proc. COMPSTAT ' 94 (Vienna), Short Communications in Computational Statistics, ed. by R. Dutter and W. Grossman, Vienna, 199-200. 
7. Glänzel, W., Telcs, A. and Schubert, A. (1984). Characterization by truncated moments and its application to Pearson-type distributions, Z. Wahrsch. Verw. Gebiete 66, 173-183.

8. Glänzel, W. and Hamedani, G.G. (2001). Characterizations of univariate continuous distributions, Studia Sci. Math. Hungar. 37, 83-118.

9. Gupta, R.C., Gupta, P.L. and Gupta, R.D. (1998). Modeling failure time data by Lehman alternatives, Commun. Statist.-Theor. Meth., 27, 887-904.

10. Gupta, R.D. and Kundu, D. (1999). Generalized exponential distribution, Austral. \& New Zealand J. Statist., 41, 173-188.

11. Gupta, R.D. and Kundu, D. (2000). Generalized exponential distribution: different method of estimations, J. Statist. Comput. Simul., 00, 1-22.

12. Gupta, R.D. and Kundu, D. (2001). Exponentiated exponential family: an alternative to gamma and Weibull distributions, Biometrical J., 43, 117-130.

13. Gupta, R.D. and Kundu, D. (2007). Generalized exponential distribution: Existing results and some recent developments, J. Statist. Plann. Inference, 137, $3537-3547$.

14. Hamedani, G.G. (1993). Characterizations of Cauchy, normal and uniform distributions, Studia Sci. Math. Hungar. 28, 243-247.

15. Hamedani, G.G. (2002). Characterizations of univariate continuous distributions. II , Studia Sci. Math. Hungar. 39, 407-424.

16. Hamedani, G.G. (2006). Characterizations of univariate continuous distributions. III , Studia Sci. Math. Hungar. 43, 361-385.

17. Hamedani, G.G. (2010). Characterizations of continuous univariate distributions based on the truncated moments of functions of order statistics, Studia Sci. Math. Hungar. 47, 462-484.

18. Hamedani, G.G. and Ahsanullah (2005). Characterizations of univariate continuous distributions based on hazard function II, JSTA, 4, 218-238.

19. Hamedani, G.G., Ahsanullah, M. and Sheng, R. (2008). Characterizations of certain continuous univariate distributions based on truncated moment of the first order statistic, Aligrah J. of Statistics, 28, 75-81.

20. Kotz, S. and Shanbhag, D.N. (1980). Some new approaches to probability distributions, Adv. in Appl. Probab. 12, 903-921.

21. Nadarajah, S. (2011). The exponentiated exponential distribution: a survey, AStA Advances in Statistical Analysis, 95 , 219-251.

22. Shakil, M. and Ahsanullah, M. (2012). Review on order statistics and record values from $F^{\alpha}$ distributions, Pak. J. Stat. Oper. Res. VIII, 101-120. 